\title{
Polymerase/nicking enzyme synergetic isothermal quadratic DNA machine and its application for one-step amplified biosensing of lead (II) ions at femtomole level and DNA methyltransferase
}

\author{
Yongxi Zhao', Feng Chen ${ }^{1}$, Qing Zhang ${ }^{1}$, Yue Zhao', Xiaolei Zuo ${ }^{2}$ and Chunhai Fan ${ }^{2}$
}

Herein, we propose a novel and universal biosensing platform based on a polymerase-nicking enzyme synergetic isothermal quadratic DNA machine (ESQM). This platform tactfully integrates two signal amplification modules, strand displacement amplification (SDA) and nicking enzyme signal amplification (NESA), into a one-step system. A bifunctional DNA probe with a stem-loop structure was designed to be partly complementary to the SDA product and digested substrate of NESA for bridging SDA and NESA. ESQM can be performed by using only the enzymes and buffer involved in the SDA module. In the presence of a target, this DNA machine is activated to afford a high quadratic amplified signal. Using $\mathrm{Pb}^{2+}$ and DNA adenine methylation (Dam) methyltransferase (MTase) as analytes, a sensitive biosensing platform is demonstrated. Low detection limits of $30 \mathrm{fM}$ $\mathrm{Pb}^{2+}$ and $0.05 \mathrm{U} \mathrm{ml}^{-1}$ Dam MTase were achieved within a short assay time $(40 \mathrm{~min})$, which were each superior to those of most previously reported methods. This DNA machine exhibited high selectivity for $\mathrm{Pb}^{2+}$. Furthermore, the successful detection of complex environmental water samples demonstrated the applicability of the proposed strategy in real samples, holding great potential for its application in environmental monitoring, biomedical research and clinical diagnosis.

NPG Asia Materials (2014) 6, e131; doi:10.1038/am.2014.84; published online 26 September 2014

\section{INTRODUCTION}

Signal amplification technologies have a critical role in molecular biology research, environmental and food monitoring, forensic identification and clinical diagnosis. Since its creation in 1985, polymerase chain reaction has become the most well-known and widely used amplification technique. ${ }^{1}$ However, this technique suffers from an intrinsic drawback, the requirement of precision thermal cycling between three different temperatures in a costly thermal cycler, limiting its popularization and application in point-of-care testing. On the other hand, various alternative isothermal amplification methods, such as rolling circle amplification (RCA), ${ }^{2,3}$ strand displacement amplification (SDA), ${ }^{4}$ loop-mediated isothermal amplification, ${ }^{5,6}$ nicking enzyme signal amplification (NESA $)^{7-9}$ and the exponential amplification reaction (EXPAR) ${ }^{10}$ have been proposed in the past two decades. These isothermal methods have been developed mainly based on some new findings in molecular biology concerning DNA synthesis and some accessory proteins together with their mimicking in vitro for nucleic acid amplification. Among these methods, EXPAR has been widely used, which is attributed to its rapid amplification kinetics (several minutes) and exceedingly high amplification efficiency $\left(10^{6}\right.$ - to $10^{9}$-fold). For example, Ye's group ${ }^{11}$ and Zhang's group ${ }^{12,13}$ have proposed several interesting methods for the highly sensitive detection of microRNA and protein based on EXPAR, respectively. Nevertheless, the EXPAR-based methods involve the weaknesses of early phase non-specific background amplification resulting from the binding of the polymerase to the single-stranded template. ${ }^{14}$ Conversely, the linear amplification strategies such as RCA, SDA and NESA are much simpler than EXPAR and out of the non-specific background amplification. Though well established and widely applied, these linear amplification strategies still face challenges in the detection of trace targets because of their relative low sensitivities.

Recently, the cascade amplification strategies, combining two or more amplification techniques together, have attracted an explosion of interest due to their ability to integrate the merits of different methods. These approaches exhibit a comparable sensitivity to the EXPAR-based methods and can avoid the high background signal caused by

\footnotetext{
${ }^{1}$ Key Laboratory of Biomedical Information Engineering of Education Ministry, School of Life Science and Technology, Xi'an Jiaotong University, Xi'an, Shaanxi, PR China and ${ }^{2}$ Division of Physical Biology, Bioimaging Center, Shanghai Synchrotron Radiation Facility, CAS Key Laboratory of Interfacial Physics and Technology, Shanghai Institute of Applied Physics, Chinese Academy of Sciences, Shanghai, PR China

Correspondence: Professor Y Zhao, Key Laboratory of Biomedical Information Engineering of Education Ministry, School of Life Science and Technology, Xi'an Jiaotong University, Xi'an, Shaanxi 710049, PR China.

E-mail:yxzhao@mail.xitu.edu.cn

or Professor C Fan, Division of Physical Biology and The Bioimaging Center, Shanghai Synchrotron Radiation Facility, CAS Key Laboratory of Interfacial Physics and Technology, Shanghai Institute of Applied Physics, Chinese Academy of Sciences, PO Box 800-204, Shanghai 201800, PR China.

E-mail: fchh@sinap.ac.cn
}

Received 26 February 2014; revised 28 May 2014; accepted 28 July 2014 
non-specific amplification. In 2008, Xie and co-workers proposed a sensitive DNA biosensor based on cascade enzymatic signal amplification by combining RCA and NESA. ${ }^{7}$ Compared with the basic NESA, this strategy significantly improved the detection sensitivity by nearly two orders of magnitude. Recently, several novel RCA-combined cascade amplification approaches have been successively developed for the high sensitive or ultrasensitive detection of nucleic acids, microRNA and cancer cells. ${ }^{15-17}$ However, all of these methods suffer from lengthy assay times (several hours) with multi-step and complex reactions. In addition, SDA was also combined with other amplification techniques to design a cascade amplification system, which is attributed to its simplicity over RCA. For instance, Willner and colleagues proposed a promising DNA machine in a one-step reaction based on integrating SDA with an $\mathrm{Mg}^{2+}$-dependent DNAzyme-assisted recycling cleavage reaction. ${ }^{18}$ Furthermore, these researchers developed an improved system that was extended to the amplified detection of cocaine. ${ }^{19}$ Unfortunately, a long time (more than $8 \mathrm{~h}$ ) was required. Moreover, the substrate for the $\mathrm{Mg}^{2+}$-dependent DNAzyme, as a signal reporter, contains a ribonucleotide that is easily degradable and requires strict operating conditions, which might cause false positive results, especially within a lengthy detection time. Recently, a twostage isothermal amplification assay was proposed by introducing SDA into NESA with a short linear quencher-fluorophore DNA probe. ${ }^{20}$ This assay improved the detection sensitivity by as much as two orders of magnitude. Nevertheless, this method was cursorily constructed by the straightforward addition of SDA and NESA, leading to a multienzyme (three enzymes) and multi-step (four steps) reaction system with a long assay time (more than $2 \mathrm{~h}$ ). Therefore, the development of one-step, rapid and simple cascade amplification strategies for ultrasensitive detection remains an urgent need.

Inspired by the dramatic finding that the reaction system of SDA contains the enzyme and buffer for the NESA reaction, herein, we construct a novel biosensor based on the polymerase-nicking enzyme synergetic isothermal quadratic DNA machine (ESQM) by integrating the SDA module with the NESA module into a one-step and simple biosensing system. ESQM can be performed in a reaction system of the basic SDA without the additional enzyme and buffer involved in NESA. In this DNA machine, a stem-loop structure bifunctional DNA probe (hairpin probe) is specially designed to be complementary with the SDA product and substrate of NESA simultaneously, acting as a 'bridge' to connect the SDA module and NESA module. Using a commercially synthesized oligonucleotide as a trigger, a high signal-tonoise ratio (18-fold) was obtained within $30 \mathrm{~min}$ using our quadratic amplification DNA machine. Then, the detection of $\mathrm{Pb}^{2+}$ was performed using ESQM combined with $\mathrm{Pb}^{2+}$-dependent GR-5 DNAzyme as the recognition probe. An ultralow detection limit down to $30 \mathrm{fM} \mathrm{Pb}^{2+}$ was achieved. Complex environmental water samples were successfully detected by the proposed biosensor. The results are in good agreement with those obtained using inductively coupled plasma mass spectrometry (ICP-MS), demonstrating the great potential of this biosensing system. Furthermore, this amplification DNA machine has also been readily expanded for the detection of DNA methyltransferase (MTase) activity and its inhibition by several anticancer or antimicrobial drugs.

\section{EXPERIMENTAL PROCEDURE}

\section{Materials and reagents}

Lead (II) acetate trihydrate and other metal salts, $\mathrm{Hg}\left(\mathrm{NO}_{3}\right)_{2}, \mathrm{MgCl}_{2}, \mathrm{MnCl}_{2}$, $\mathrm{CaCl}_{2}, \mathrm{SnCl}_{2}, \mathrm{FeCl}_{3}, \mathrm{CdCl}_{2}, \mathrm{CoCl}_{2}$ and $\mathrm{ZnCl}_{2}$, were purchased from SigmaAldrich (St Louis, MO, USA). Klenow fragment $\left(\mathrm{exo}^{-}\right)$, Nt.BbvCI, DNA adenine methylation (Dam) MTase and the corresponding buffers were obtained from New England Biolabs Ltd. (Beijing, China). All the oligonucleotides were synthesized by Sangon Biotechnology Co., Ltd. (Shanghai, China). The sequences are listed in Supplementary Table S1. All the other solutions were prepared and diluted with Milli-Q water (resistance >18.2 M $\Omega$ ).

\section{$\mathrm{Pb}^{2+}$ detection based on ESQM}

To detect $\mathrm{Pb}^{2+}$, various concentrations of $\mathrm{Pb}^{2+}$ were added to $20 \mu \mathrm{l}$ of $1 \times$ NEBuffer 2 containing $10 \mathrm{~nm}$ DNAzyme, $10 \mathrm{~nm}$ SDA template, $50 \mathrm{~nm}$ hairpin probe, $250 \mathrm{~nm}$ molecular beacon (MB), 2.5 units of Klenow Fragment, 0.8 units of Nt.BbvCI and $0.2 \mathrm{~mm}$ dNTPs. Subsequently, the solutions were incubated at $37^{\circ} \mathrm{C}$ for $40 \mathrm{~min}$ before the fluorescence measurement. The river water samples were first filtered through a $0.2-\mu \mathrm{m}$ membrane to remove the insoluble substance and were then treated by $5 \% \mathrm{HNO}_{3}$ and $10 \mathrm{~mm} \mathrm{Mg}{ }^{2+}$ before testing under the same conditions described above. A Thermo X Series 2 ICP-MS (Thermo Fisher Scientific, Shanghai, China) was used in this work.

\section{Application for Dam MTase activity assay}

The experiments were performed in $20 \mu \mathrm{l} 1 \times$ NEBuffer 2 containing a $25-\mathrm{nm}$ Dam-probe, $10 \mathrm{~nm}$ SDA template, $50 \mathrm{~nm}$ hairpin probe, $250 \mathrm{~nm} \mathrm{MB,} 160 \mu \mathrm{M}$ SAM, 2 units of DpnI, $0.2 \mathrm{~mm}$ dNTPs, 0.8 units of Nt.BbvCI and 2.5 units of Klenow Fragment. After the addition of various concentrations of Dam MTase, the solutions were incubated at $37^{\circ} \mathrm{C}$ for $40 \mathrm{~min}$. Then, fluorescence emission spectra were recorded as described above.

\section{RESULTS AND DISCUSSION}

\section{Principle of ESQM-based biosensor}

The principle of the ESQM-based biosensor is outlined in Figure 1. Three main components, SDA template, bifunctional hairpin probe and $\mathrm{MB}$ (digested substrate of NESA) are employed. The SDA template consists of a primer-binding domain (P', blue), an amplification domain ( $\mathrm{X}$ ', red violet) and a recognition sequence ( $\mathrm{R}^{\prime}$, yellow) for nicking enzyme Nt.BbvCI that recognizes double-strand DNA and cleaves only one strand. The bifunctional hairpin probe containing a stem-loop structure is specially designed to be partly complementary to the SDA product and digested substrate of NESA, respectively. This probe is used as a 'bridge' to connect the SDA module and NESA

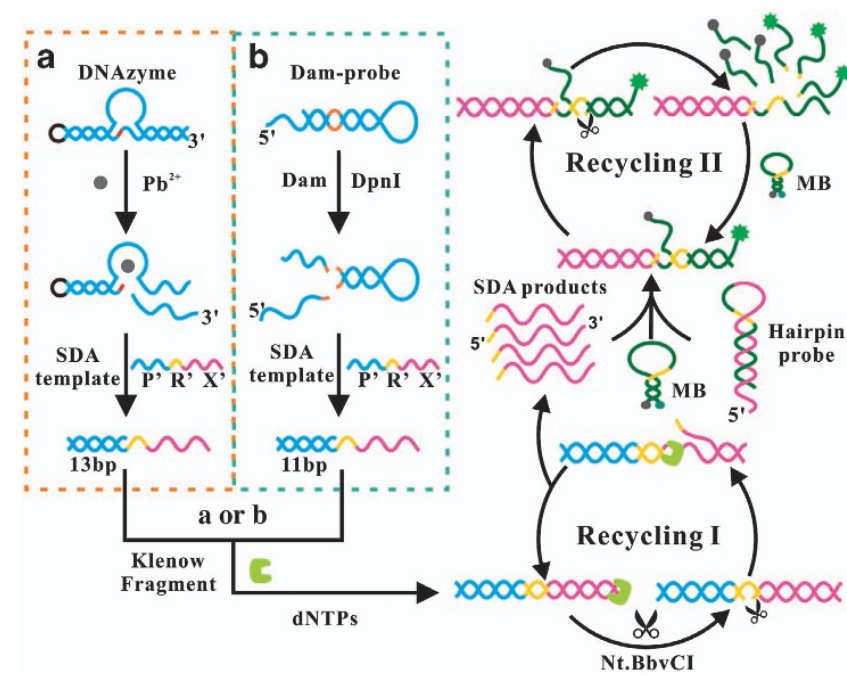

Figure 1 Schematic illustration of ESQM and its application for $\mathrm{Pb}^{2+}$ and Dam MTase detection. (a) $\mathrm{Pb}^{2+}$ activates GR-5 DNAzyme for the cleavage reaction at $\mathrm{rA}$ (highlighted in red), which generates a primer to trigger ESQM. (b) ESQM is initiated by the primer produced via the Dam MTase and Dpnl-coupled reaction. Recycling I shows the SDA process of ESQM and Recycling II depicts the NESA process induced by SDA products and the hairpin probe, leading to a quadratic amplified signal. 
module. As the digested substrate of NESA, the MB-containing recognition sequence for $\mathrm{Nt}$.BbvCI also serves as a signal unit and is designed to be a stem-loop structure labeled with a fluorophore and quencher at its 5' and 3' end, respectively. The fluorescence signal is quenched due to the fluorescence resonance energy transfer reaction between the fluorophore and the quencher.

When the target is present (A or B), ESQM is activated. In detail, the extension reaction in the SDA module is initiated with the help of the DNA polymerase Klenow Fragment and dNTPs, producing the double-stranded SDA template containing the full recognition site for Nt.BbvCI. Following the nicking reaction by Nt.BbvCI, a new extension will continue with the displacement and release of the single-strand (ss) DNA SDA product. In this way (Recycling I), thousands of SDA products are generated, which open the hairpin probe with complementary hybridization as the trigger for NESA. Then, the opened-hairpin probe can hybridize to $\mathrm{MB}$, forming a DNA duplex with the full recognition site for Nt.BbvCI. After the nicking reaction of $\mathrm{MB}$, the separation of the fluorophore from the quencher occurs as well as the release of the opened-hairpin probe. The disassociated opened-hairpin probe can hybridize with another uncleaved $\mathrm{MB}$ and trigger the second cycle of nicking, leading to an amplified detection signal (Recycling II). Eventually, each target can undergo a quadratic amplification signal by ESQM. Note that the sensing process is performed in a one-step reaction at $37^{\circ} \mathrm{C}$. Furthermore, the nicking enzyme and reaction buffer involved in SDA are also suitable for NESA. All of these merits are superior to those of some reported methods. ${ }^{7,17,20}$

In theory, we can calculate the recycling process of the linear and quadratic amplification system as detailed in Supplementary Table S2. Their signal equations are described as $Y_{1}=N$ for the linear amplification mode (basic SDA, see Supplementary Figure S1) and $Y_{2}=0.5 N(N-1)$ for the quadratic amplification mode (ESQM), where $Y_{1}$ and $Y_{2}$ represent the signal of the linear and quadratic amplification mode, respectively, and $N$ is the positive integer. $Y_{2}$ is always greater than $Y_{1}$ when $N>3$ (meaning a sufficient reaction time). To confirm the amplification efficiency of our proposed DNA machine, a series of experiments was performed for comparison with the basic SDA without the integration of the NESA module. As demonstrated in the inset of Figure 2, the background fluorescence intensity of ESQM in the absence of SDA primer (curve c) slightly increased compared with that of the basic SDA (curve d). This result should be due to the damage of the stem-loop structure of MB by partial hybridization with the hairpin probe. Fortunately, when the SDA primer $(10 \mathrm{nM})$ is present, the fluorescence signal of ESQM (curve a) is much higher than that of the basic SDA process (curve b). A high signal enhancement of 18.0-fold was observed in the quadratic amplified strategy, whereas only a 10.1-fold signal increase was achieved by the basic SDA. These results, in accordance with our above theoretical analysis, indicate that the proposed DNA machine can provide a higher amplified signal by integrating the SDA module with the NESA module.

\section{Optimization of sensing conditions}

As the 'bridge' between the SDA module and NESA module, the hairpin probe is specifically designed in our strategy. Its structure is very important for the sensing performance. In detail, this probe should be easily opened by the SDA product and hybridize with MB to trigger the NESA process rapidly in the presence of a target. Conversely, when the target is absent, the hairpin probe should be stabilized and fail to hybridize with $\mathrm{MB}$ because of the strong intramolecular hybridization with the long stem, yielding a low background signal. To obtain the best sensing performance, the stem length of the hairpin probe was investigated. As presented in Supplementary Table S1, there are three different hairpin probes (H1, H2 and H3), each including $8 \mathrm{bp}, 10 \mathrm{bp}$ and 12-bp in the stem region. Their potential secondary structures and the corresponding Gibbs-free energy are shown in Supplementary Figure S2. Their fluorescence responses are shown in Supplementary Figure S3. In the presence of a target, decreased fluorescence intensity was observed with the lengthening of the stem, which revealed that the hairpin probe with short stem can be more easily opened by the SDA product to produce a higher signal. Unfortunately, higher background fluorescence also occurred with the shorter stem hairpin probe, mainly due to the competing hybridization between $\mathrm{MB}$ and the hairpin probe. While $\mathrm{H} 1$ provided the highest fluorescence intensity, $\mathrm{H} 2$ was used in the experiments because of its highest signal-to-noise ratio. Subsequently, the molar ratio of the hairpin probe to $\mathrm{MB}$ (concentration is fixed at $250 \mathrm{~nm}$ ) and the enzyme concentrations were optimized. The results are presented in Figures $3 \mathrm{a}-\mathrm{c}$. On the basis of the highest $F / F_{0}$ value, a 1:5 molar ratio of the hairpin probe to $\mathrm{MB}$, 0.8 units of Nt.BbvCI and 2.5 units of Klenow Fragment were employed for the following experiments.

In addition, the effect of the reaction time was also investigated. As depicted in Figure 3d, with the prolongation of the reaction time, the fluorescence intensity in the presence of the SDA primer rapidly increased, indicating that the recycling cleavage of MB by the quadratic amplification integrating SDA with NESA was triggered. The intensity nearly approached steady state after incubation for $50 \mathrm{~min}$. Note that a slow fluorescence enhancement was also observed in the absence of the SDA primer. This background fluorescence signal mainly results from the competing hybridization between the hairpin probe and $M B$, which damaged the stem-loop structure of $\mathrm{MB}$ to produce a fluorescence signal. To obtain the best sensing performance, $30 \mathrm{~min}$ was selected because of the highest fluorescence enhancement. This assay time and the high signal-to-noise ratio (18-fold) are superior to those of the previously reported methods based on cascade amplification. $^{21-25}$

\section{Analytical performance for $\mathrm{Pb}^{2+}$ detection based on ESQM}

For the detection of $\mathrm{Pb}^{2+}$, a DNAzyme (GR-5 DNAzyme) is involved in the proposed biosensor as a $\mathrm{Pb}^{2+}$ recognition unit. GR-5 DNAzyme is a functionalized nucleic acid with catalytic activity, cleaving the

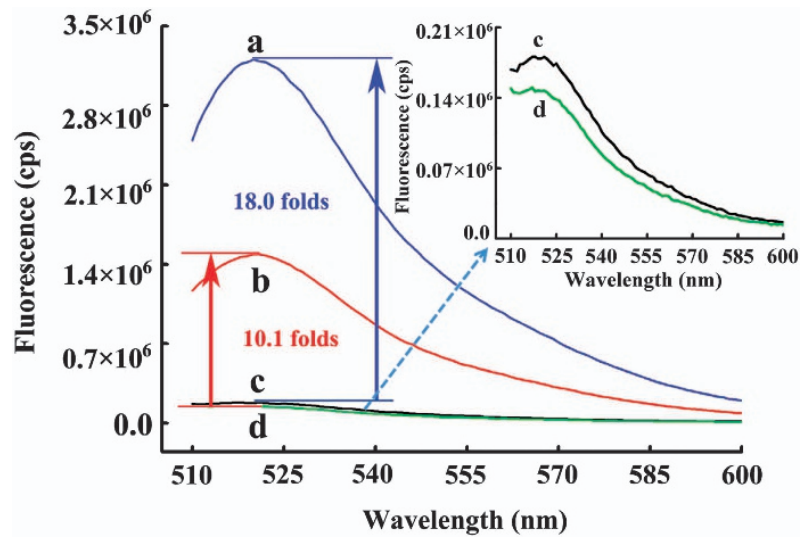

Figure 2 The fluorescence responses of the proposed sensing system (a) and basic SDA system (b) in the presence of $10 \mathrm{~nm}$ SDA primer, with corresponding backgrounds without SDA primer (c, d), respectively. Inset: Fluorescence spectra of (c) and (d). 

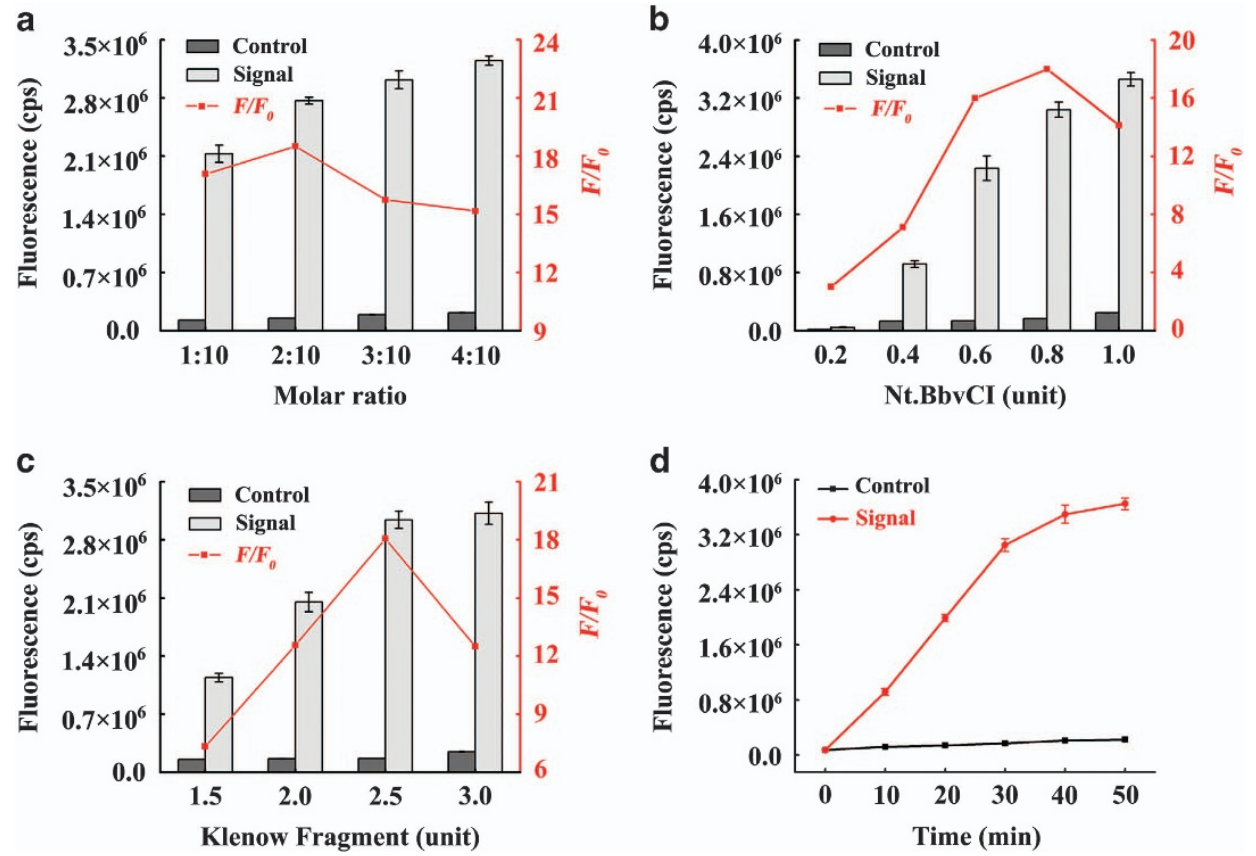

Figure 3 Optimization of the experimental conditions. The effect of the molar ratio of the hairpin probe to $\mathrm{MB}$ (a), $\mathrm{Nt}$. BbvCl concentration (b) and $\mathrm{Klenow}$ Fragment concentration (c) on the fluorescence response of the DNA machine. $F_{0}$ and $F$ represent the fluorescence intensity in the absence and presence of $10 \mathrm{~nm}$ SDA primer, respectively. (d) The time-dependent fluorescence response with $10 \mathrm{~nm}$ SDA primer over background fluorescence. The error bar indicates the standard deviation of three independent experiments.

oligonucleotide substrate at the ribonucleotide (rA) recognition position in the presence of $\mathrm{Pb}^{2+}$. In the absence of $\mathrm{Pb}^{2+}$, the GR-5 DNAzyme exhibits no activity, and the substrate strand of DNAzyme is not cleaved. There is no primer existing to trigger the DNA machine. Because Nt.BbvCI is disabled to cleave the ssDNA, no fluorescence enhancement is observed. However, when $\mathrm{Pb}^{2+}$ is present, the recognition unit GR-5 DNAzyme performs the catalytic reaction for the hydrolytic cleavage of the substrate strand at $\mathrm{rA}$ (Figure 1a). Due to the low $T_{\mathrm{m}}\left(\sim 26^{\circ} \mathrm{C}\right)$, an ssDNA (13 mer) is released at the reaction temperature $\left(37^{\circ} \mathrm{C}\right)$. Then, the released ssDNA hybridizes with the SDA template and acts as a primer to initiate the SDA process in the assistance of DNA polymerase and dNTPs. Finally, each $\mathrm{Pb}^{2+}$ can induce a quadratic amplified signal by ESQM as discussed above.

To obtain the best sensing performance, several important assay conditions including the concentration of DNAzyme and the reaction time were studied, and the results are presented in Figure 4. On the basis of the highest value of $F / F_{0}, 10 \mathrm{~nm}$ DNAzyme and a 40-min reaction time were selected. Under the optimized conditions, gelelectrophoresis experiments were employed to demonstrate $\mathrm{Pb}^{2+}$ detection by the developed DNA machine. As shown in Figure 5a, no new product of cleaved MB was observed (lane a, in the absence of $\left.\mathrm{Pb}^{2+}\right)$. However, new bands of the cleaved MB were obviously present in the presence of $\mathrm{Pb}^{2+}$ (lane $\mathrm{b}$ ), suggesting that $\mathrm{Pb}^{2+}$-triggered quadratic amplification occurred. Then, a series of samples containing various $\mathrm{Pb}^{2+}$ concentrations were examined to evaluate the sensing performance of our sensing system. Figure $5 \mathrm{~b}$ presents the fluorescence spectra in the presence of $\mathrm{Pb}^{2+}$ concentrations ranging from 0 to $50 \mathrm{~nm}$. As observed, the fluorescence intensity gradually increased when the $\mathrm{Pb}^{2+}$ concentration was increased. With a correlation coefficient of $R^{2}=0.9985$, a linear relationship between the fluorescence intensity $(518 \mathrm{~nm})$ and logarithm of the $\mathrm{Pb}^{2+}$ concentration is achieved (inset, Figure 5c). With a large dynamic range (6 orders of magnitude), a detection limit down to $30 \mathrm{fM}$ was obtained based on three times standard deviation of the blank, which is at least 500 -fold lower than those of previously reported fluorescence methods. ${ }^{22,26-29}$ This sensitivity is also much higher than those of several electrochemical and electrochemiluminescence approaches ${ }^{24,25,30,31}$ and is even comparable to the surface plasmon resonance-based biosensor. ${ }^{32}$ The details are summarized in Table 1.

Control experiments with $\mathrm{Pb}^{2+}$ at various concentrations were also performed in the system only including basic SDA without the NESA module (see Supplementary Figure S1). As demonstrated in Supplementary Figure S4, a detection limit of only $25 \mathrm{pM}$ was obtained. This sensitivity is approximately three orders of magnitude lower than that of the proposed DNA machine integrating the SDA module with the NESA module. Therefore, all these results revealed that a quadratic amplified signal and ultrahigh sensitivity for $\mathrm{Pb}^{2+}$ detection can be achieved with the amplified detection platform based on ESQM.

Selectivity was another important issue for the $\mathrm{Pb}^{2+}$ detection. Other environmentally relevant metal ions $\left(\mathrm{Zn}^{2+}, \mathrm{Co}^{2+}, \mathrm{Ca}^{2+}, \mathrm{Hg}^{2+}\right.$, $\mathrm{Cd}^{2+}, \mathrm{Fe}^{3+}, \mathrm{Mn}^{2+}, \mathrm{Sn}^{2+}$ and $\mathrm{Mg}^{2+}$ ) were selected to investigate the selectivity of this assay. The fluorescent response of $\mathrm{Pb}^{2+}$ $(10 \mathrm{~nm})$ and other metal ions $(100 \mathrm{~nm})$ is shown in Figure $5 \mathrm{~d}$. Only $\mathrm{Pb}^{2+}$ triggered a significant fluorescence enhancement, whereas a very little increase in the fluorescent intensity was observed in the presence of the other interference ions. This finding indicated that the ESQM-based biosensing system exhibits a good specificity to $\mathrm{Pb}^{2+}$ over other competing metal ions. The excellent selectivity is due to the GR-5 DNAzyme, which is $\sim 40000$ times more selective for $\mathrm{Pb}^{2+}$ against the most active interfering metal ion $\mathrm{Zn}^{2+} .33$ The 8-17 DNAzyme, another DNAzyme used for most $\mathrm{Pb}^{2+}$ biosensors, exhibits at most 160-fold selectivity against $\mathrm{Zn}^{2+} .33$ 
$\mathrm{Pb}^{2+}$ detection of water sample from Xi'an Chan River

The maximum contamination level of $\mathrm{Pb}^{2+}$ in drinking water defined by the US Environmental Protection Agency is $72 \mathrm{nM}$, which is $\sim 10^{6}$
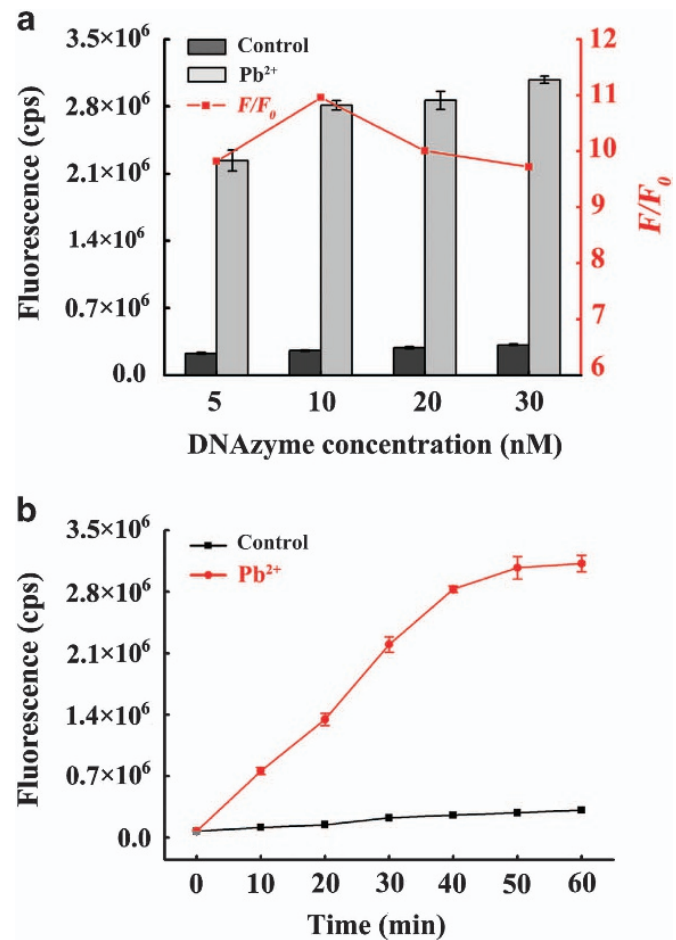

Figure 4 Optimization of DNAzyme concentration (a) and reaction time (b) for $\mathrm{Pb}^{2+}$ detection. fold higher than the detection limit of our sensing system. This requirement indicates that the proposed sensing system holds great potential for real water sample analysis of $\mathrm{Pb}^{2+}$. Acidification pretreatment of complex environmental samples is always used to reduce interference by organic matter and to convert metals associated with particulates to a form (usually a free metal) that can be detected by atomic absorption spectroscopy or ICP. Inspired by this procedure, the filtered river water was treated with an acidification process before analysis. Nitric acid was used due to the adequate digestion of most samples. As illustrated in Figure 6a, no obvious signal responses were observed for the ultrapure water samples (negative controls) treated with nitric acid by our biosensor. However, the filtered river water samples undergoing the acidification process exhibited significant $\mathrm{Pb}^{2}$ ${ }^{+}$-dependent signal responses. In addition, the 5\%(w/v) nitric acid resulted in the highest fluorescence intensity. Note that these water samples were diluted for the detection using our biosensor. The acidification time was also investigated, as depicted in Supplementary Figure S5A, and $1 \mathrm{~h}$ was selected as the signal reached a plateau effect. The above result of nitric acid-triggered $\mathrm{Pb}^{2+}$ 'output' in the filtered river water may be attributed to the conversion of lead associated with particulate to free $\mathrm{Pb}^{2+}$ and the release of free $\mathrm{Pb}^{2+}$ form $\mathrm{Pb}^{2+}$ coordination compounds under the acidic condition. Note that the GR-5 DNAzyme is selective for only free $\mathrm{Pb}^{2+}$ rather than other forms, such as $\mathrm{Pb}^{2+}$ coordination compounds. Furthermore, considering the dynamic equilibrium of the complexation reaction, we hypothesize that adding an overbalance of other metal ions as competitors may be helpful in displacing $\mathrm{Pb}^{2+}$ from its coordination compounds. $\mathrm{Mg}^{2+}$, acting as a cofactor for most enzymes, is employed as a model competitor. As illustrated in Figure 6b, the introduction of a high $\mathrm{Mg}^{2}$ + concentration (mM level) to the filtered river water leads to an
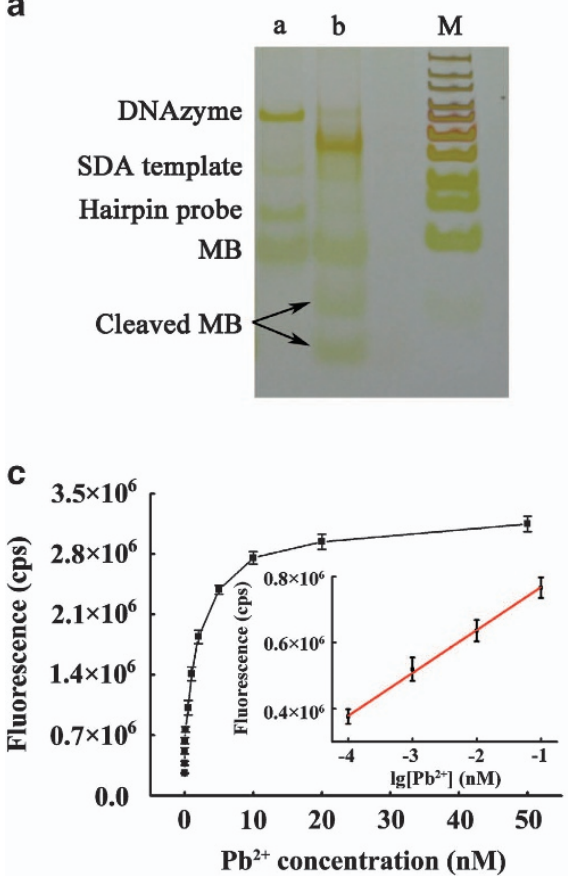

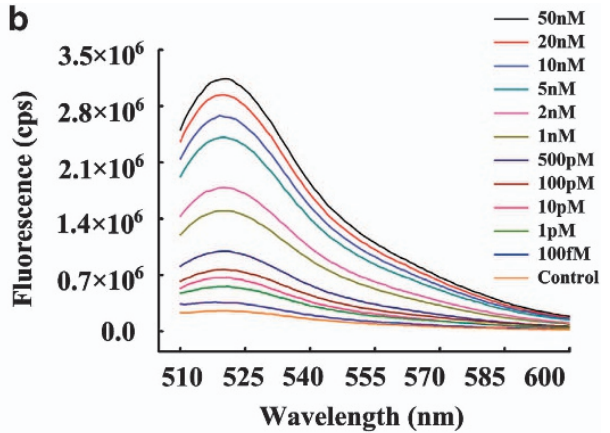

d

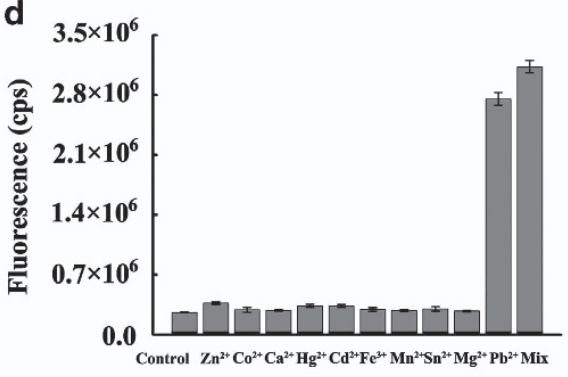

Figure 5 (a) Non-denaturing PAGE analysis. Lane M, marker; lane a, $\mathrm{Pb}^{2+}$ detection by ESQM in the absence of $\mathrm{Pb}^{2+}$; lane b, $\mathrm{Pb}^{2+}$ detection by ESQM with $20 \mathrm{nM} \mathrm{Pb}^{2+}$. (b) Fluorescence spectra response to different concentrations of $\mathrm{Pb}^{2+}$. (c) Changes in fluorescence intensity at $518 \mathrm{~nm}$ upon the addition of various concentrations of $\mathrm{Pb}^{2+}$. The inset displays the linear correlation between the fluorescence intensity and the logarithm of the $\mathrm{Pb}^{2+}$ concentration in the range of $100 \mathrm{fM}$ to $100 \mathrm{pM}$. (d) Selectivity of the sensing strategy for $\mathrm{Pb}^{2+}$ detection over other common interference metal ions and the mixture of these metal ions. The concentration of $\mathrm{Pb}^{2+}$ is $10 \mathrm{~nm}$, whereas the concentrations of other metal ions are each $100 \mathrm{~nm}$. 
Table 1 Comparison of $\mathrm{Pb}^{2+}$ detection methods

\begin{tabular}{|c|c|c|c|c|}
\hline Method & Detection limit ( $p M)$ & Assay time (min) & Steps & Reference \\
\hline Fluorescence detection based on ESAM & 0.03 & 40 & One step & Current method \\
\hline Fluorescence detection based on lead(II)-driven DNA molecular device & 20000 & 120 & One step & 26 \\
\hline Amplified fluorescence detection by graphene and DNAzyme & 300 & 20 & One step & 27 \\
\hline Amplified fluorescence detection based on catalytic MB & 600 & 10 & One step & 28 \\
\hline Amplified fluorescence detection by exonuclease-aided DNA recycling & 5 & 150 & Two steps & 22 \\
\hline Amplified fluorescence detection by isothermal strand displacement signal amplification & 200 & 195 & Multi-steps & 29 \\
\hline Enzyme-free strip biosensor based on catalytic DNA circuit & 10 & 105 & Three steps & 23 \\
\hline Amplified electrochemical assay by hybridization chain reaction & 37 & 200 & Three steps & 24 \\
\hline ECL detection using DNAzyme & 0.11 & Not mentioned & One step & 30 \\
\hline Electrochemical biosensor based on DNA functionalized gold nanoparticles & 28 & 780 & Three steps & 31 \\
\hline Amplified electrochemical detection based on RCA & 7.8 & 365 & Multi-steps & 25 \\
\hline Amplified SPR detection using gold nanoparticles & 0.005 & 100 & One step & 32 \\
\hline
\end{tabular}

Abbreviations: ECL, electrochemiluminescence; RCA, rolling circle amplification; SPR, surface plasmon resonance.
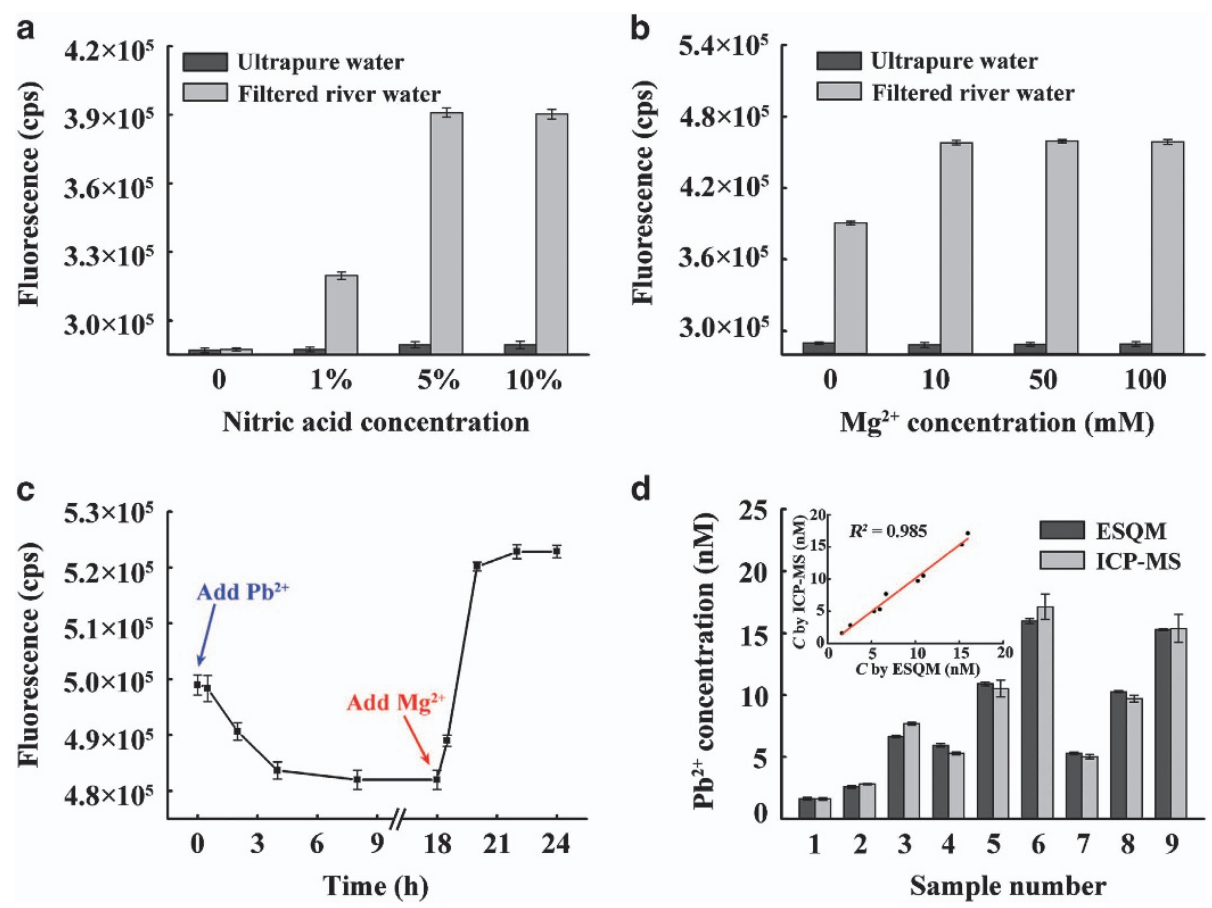

Figure 6 Performance of the proposed biosensors in the analysis of environmental water samples. (a) The concentration-dependent effect of nitric acid on the $\mathrm{Pb}^{2+}$-triggered signal response. (b) The concentration-dependent effect of $\mathrm{Mg}^{2+}$ on the $\mathrm{Pb}^{2+}$-triggered signal response. (c) Time-dependent $\mathrm{Pb}{ }^{2+}$ complexation and displacement in river water. (d) Determination of $\mathrm{Pb}^{2+}$ in environmental water samples using the proposed ESQM biosensor and ICP-MS. Samples 1-9 represent drinking water samples spiked with 0,1 and $5 \mathrm{~nm} \mathrm{~Pb}^{2+}$, Chan River water samples spiked with 0,5 and $10 \mathrm{~nm} \mathrm{~Pb}^{2+}$ and Ba River samples spiked with 0,5 and $10 \mathrm{~nm} \mathrm{~Pb}^{2+}$, respectively. The inset shows the strong correlation between these two methods with a correlation coefficient of 0.985 . $C$ represents the $\mathrm{Pb}^{2+}$ concentration.

increased $\mathrm{Pb}^{2+}$-dependent signal response, demonstrating our above hypothesis. In addition, the time of the $\mathrm{Mg}^{2+}$ process was also optimized (Supplementary Figure S5B). To verify the complexation of $\mathrm{Pb}^{2+}$ in the river water sample, filtered river water without acid or the $\mathrm{Mg}^{2+}$ process was spiked with a standard $\mathrm{Pb}^{2+}$ concentration. Time-dependent tests of this sample were performed after the addition of $\mathrm{Pb}^{2+}$. Figure $6 \mathrm{c}$ reveals that the signal of $\mathrm{Pb}^{2+}$ decreases with time, implying the complexation of the added $\mathrm{Pb}^{2+}$ by the complexing agent existing in the river water. Nevertheless, after adding excess $\mathrm{Mg}^{2+}$ to the same sample, the $\mathrm{Pb}^{2+}$-triggered signal increases and is ultimately higher than that of the spiked $\mathrm{Pb}^{2+}$ concentration, indicating the successful displacement of both spiked $\mathrm{Pb}^{2+}$ and intrinsic $\mathrm{Pb}^{2+}$ from the coordination compounds.

Under the optimized condition of the nitric acid and $\mathrm{Mg}^{2+}$ process, a linear relationship between the logarithm of the $\mathrm{Pb}^{2+}$ concentration $(0.1-100 \mathrm{pM})$ and fluorescence intensity is achieved, with the corresponding linear equation of $I_{\mathrm{F}}=1.47 \lg \left[\mathrm{Pb}^{2+}\right]+4.67$, where $I_{\mathrm{F}}$ represents the fluorescence intensity $\left(\times 10^{5} \mathrm{cps}\right)$. Then, filtered water samples from the Chan River treated with nitric acid and $\mathrm{Mg}^{2+}$ were spiked with 0,5 and $10 \mathrm{nM} \mathrm{Pb}^{2+}$ and tested using the proposed ESQM biosensor. As depicted in Figure $6 \mathrm{~d}$, the $\mathrm{Pb}^{2+}$ concentration in the Chan River water is $5.95 \mathrm{nM}$, as detected by ESQM. Satisfactory recoveries of 98.5 and $102.12 \%$ were obtained for the detection of 
these spiked samples. These samples were also tested by ICP-MS to validate the $\mathrm{Pb}^{2+}$ concentrations, the result of which is presented in Figure $6 \mathrm{~d}$ and the inset. The determined $\mathrm{Pb}^{2+}$ concentrations of the Chan River filtered water samples are consistent with the results obtained using our ESQM biosensor (correlation coefficient of 0.985). To further confirm the practical application of our biosensor, water samples from another river (the Ba River) (7-9 samples, Figure 6d) and drinking water samples (1-3 samples, Figure 6d) were also tested using our ESQM biosensor and ICP-MS with satisfactory results. These results clearly demonstrate that our proposed biosensor is capable of detecting $\mathrm{Pb}^{2+}$ in environmental water samples with excellent performance. In addition, the high sensitivity results in a high dilution factor for complex samples, which is significant for the decrease or even elimination of possible interference from different background compositions in these samples.

\section{Application of ESQM for the detection of DNA MTase}

To demonstrate the versatility of the proposed strategy, we applied the developed DNA machine to construct another detection system for the assay of DNA MTase which is closely related to human disease such as cancer. ${ }^{34,35}$ Herein, Dam MTase, a representative DNA MTase, was used as the model target. As illustrated in Figure 1b, a stem-loop structure Dam probe with a long stem was employed as the recognition unit for Dam MTase detection. In the presence of Dam MTase, the Dam probe is methylated, forming the substrate for DpnI. Subsequently, the cleavage of methylated Dam probe results in the release of a short ssDNA (11 mer) at the reaction temperature. This ssDNA is complementary to the SDA template, which can serve as an SDA primer to activate the DNA machine and generate an amplified fluorescence signal, as discussed above. Conversely, when Dam MTase is absent, $D p n \mathrm{I}$ cannot cut the Dam probe. The double helical structure of the Dam probe is maintained, and no primer is produced
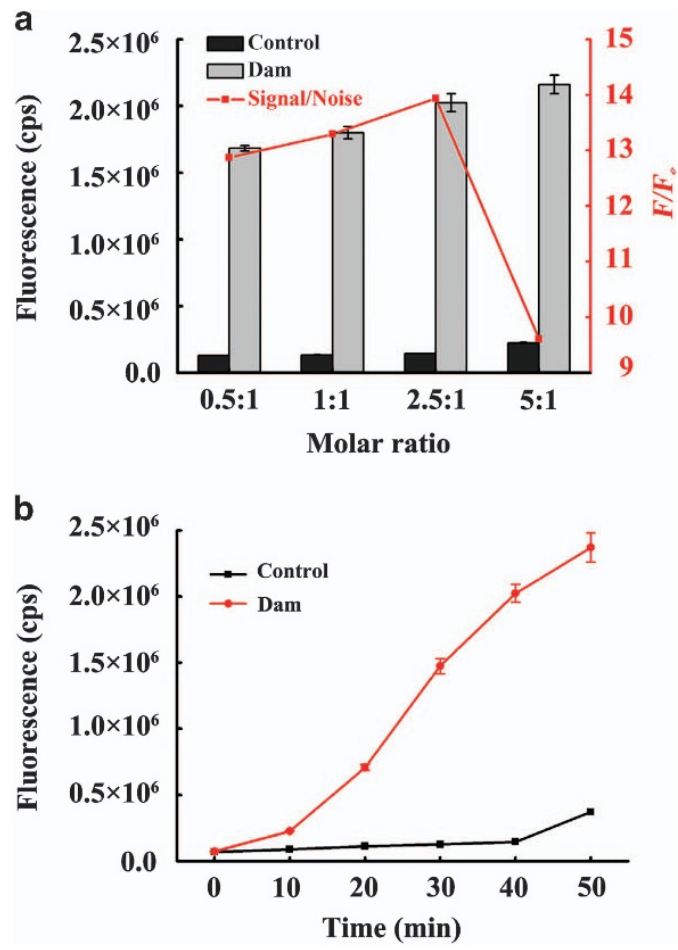

Figure 7 Effect of Dam probe concentration (a) and reaction time (b) on the performance of the Dam MTase assay. to trigger the DNA machine. Eventually, no obvious fluorescence signal is observed. Note that ESQM can be readily expanded for the detection of many other analytes, such as miRNA, ATP, cocaine and thrombin, with minor modification. For instance, only a new template is required for target miRNA to trigger ESQM. To design the sensing system for ATP, cocaine or thrombin, their aptamers are needed for target recognition and the trigger of ESQM. In all of these cases, the hairpin probe and $\mathrm{MB}$ do not need to be changed.

To obtain the best sensing performance, several conditions including the Dam probe concentration and reaction time were studied, the results of which are presented in Figure 7. It can be observed that both the 25-nM Dam probe and $40 \mathrm{~min}$ resulted in the highest signal-tonoise ratio, and these conditions were selected for the following experiments. Under these optimized assay conditions, various concentrations of Dam MTase were introduced, and the fluorescence spectra are shown in Figure 8a. The fluorescence intensity increased continuously with increasing Dam MTase concentration. Figure $8 \mathrm{~b}$ shows the linear relationship between the fluorescence intensity and the Dam MTase concentration between 0.4 and $4 \mathrm{U} \mathrm{ml}^{-1}$. A low detection limit of $0.05 \mathrm{U} \mathrm{ml}^{-1}$ was achieved, which is superior to that of most previously reported methods. ${ }^{36-38}$ In addition, the high selectivity for Dam MTase against other enzymes, including M.SssI MTase and DNA ligase, was successfully demonstrated (Supplementary Figure S6).

Numerous studies have demonstrated that Dam MTase has a vital role in several human diseases and bacterial virulence; thus, the evaluation of Dam MTase inhibition might contribute to important applications in antimicrobial drug development. In this work, several antimicrobial drugs were employed to investigate the inhibition of Dam MTase. The sensing system contains another three enzymes
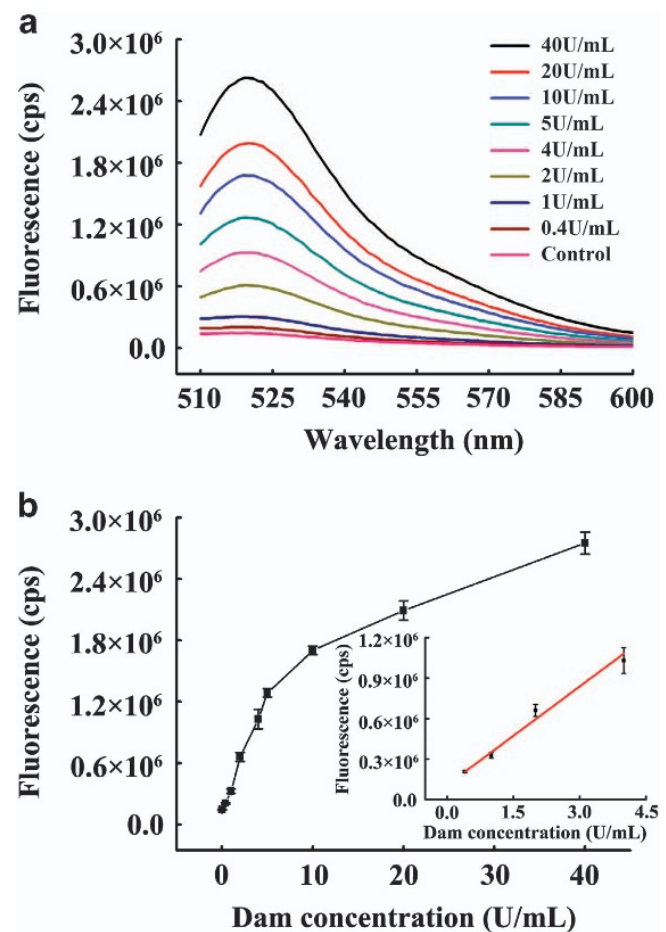

Figure 8 (a) Fluorescence spectra response to various concentrations of Dam MTase. (b) Changes in fluorescence intensity at $518 \mathrm{~nm}$ upon the addition of various Dam MTase concentrations. The inset displays the linear correlation between the fluorescence intensity and Dam MTase concentration. 

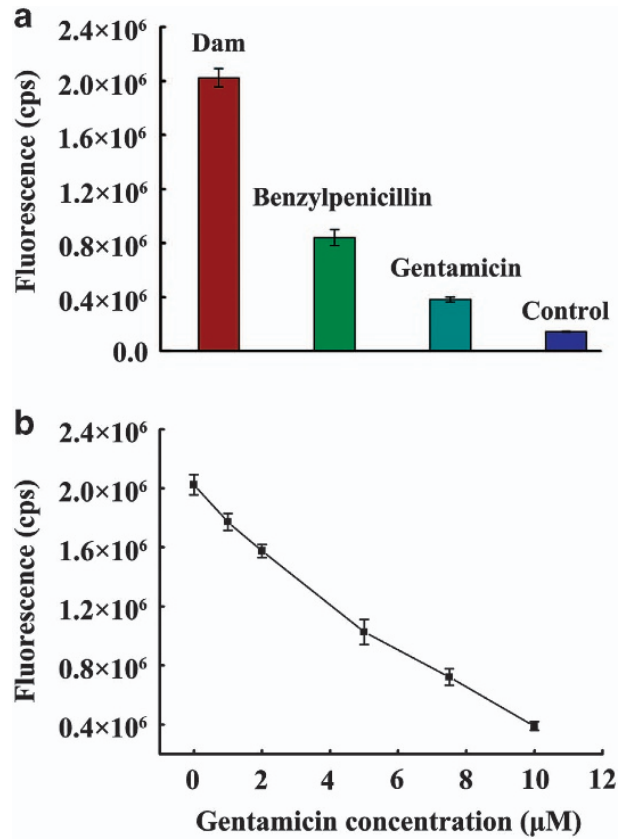

Figure 9 (a) Effect of benzylpenicillin $(10 \mu \mathrm{m})$ and gentamycin $(10 \mu \mathrm{m})$ on the activity of Dam MTase. (b) Inhibition of different concentrations of gentamycin on Dam MTase activity. The gentamycin concentrations were 0 , $1,2,5,7.5$ and $10 \mu \mathrm{m}$.

(DpnI, Nt.BbvCI and Klenow Fragment); thus, control experiments (details are provided in Supplementary Information) were first performed to determine whether these drugs affect their activities. As depicted in Supplementary Figure S7, no obvious changes in the fluorescence intensity were observed in the absence and presence of these inhibitors, indicating that these drugs have no effect on their activities for concentrations of $10 \mu \mathrm{M}$. Thus, these concentrations of different inhibitors were used to investigate the inhibition of the Dam MTase activity. The fluorescence intensity in response to the inhibitors is shown in Figure 9a, and both inhibitors exhibited a significant inhibitory effect on Dam MTase. In addition, we further assessed the concentration-dependent inhibition of the Dam MTase activity using various gentamycin concentrations. Figure $9 \mathrm{~b}$ demonstrates that the inhibition was gradually enhanced with increasing gentamycin concentration. Therefore, the proposed fluorescent strategy holds the potential to be a promising platform for the screening of antimicrobial drugs.

\section{CONCLUSIONS}

In summary, we have developed a novel and universal biosensing platform based on the ESQM, integrating an SDA module and an NESA module into a one-step and simple sensing system. A bifunctional hairpin probe was specially designed as a bridge between these two isothermal signal amplification modules. This DNA machine was effectively implemented under the basic SDA reaction conditions without the introduction of additional enzymes or a complex reaction buffer. Using $\mathrm{Pb}^{2+}$ as a model analyte, ultrasensitive detection with a detection limit of $30 \mathrm{fM}$ was demonstrated in a short assay time ( $40 \mathrm{~min}$ ), which is three orders of magnitude lower than that of a basic SDA assay. This sensitivity is also much higher than those of most reported fluorescence and electrochemical biosensors. Moreover, an excellent selectivity for $\mathrm{Pb}^{2+}$ was achieved even in the presence of various interferential metal ions with high concentrations. The satisfactory performance on river and drinking water samples demonstrated the potential applications of the proposed biosensor in complex environmental water samples. The versatility of the proposed sensing platform was also demonstrated by the successful detection of DNA MTase activity and its inhibition. As a universal sensing system, this DNA machine can be readily constructed to be an electrochemical or electrochemiluminescence biosensor by only changing the signal unit ( $\mathrm{MB}$ in this work) to corresponding signal reporters (electrochemical $\mathrm{MB}$ or electrochemiluminescence MB). ${ }^{39,40}$ Our ongoing work will demonstrate the applicability of this ESQM-based electrochemical biosensor combined with DNA nanostructure in the detection of cancer biomarkers such as microRNA and DNA methylation.

\section{CONFLICT OF INTEREST}

The authors declare no conflict of interest.

\section{ACKNOWLEDGEMENTS}

This research was financially supported by the National Basic Research Program 973 of China (No. 2013CB933800, 2012CB932600, 2011CB707903 and 2010CB732603), the Natural Science Foundation of Shaanxi Province (No. 2013JQ2017), the Fundamental Research Funds for the Central Universities (xjj2014130) and the Research Project Foundation of the Shaanxi Branch of the China National Tobacco Corporation (No. 201112029). We would like to thank Assistant Engineer He Wu at the Shaanxi Bureau of Geology and Mineral Resources (Xi'an, Shaanxi) for supporting the ICP-MS analysis.

1 Saiki, R. K. Scharf, S., Faloona, F., Mullis, K. B., Horn, G. T., Erlich, H. A. \& Arnheim, $\mathrm{N}$. Enzymatic amplification of beta-globin genomic sequences and restriction site analysis for diagnosis of sickle cell anemia. Science 230, 1350-1354 (1985).

2 Zhao, Y., Qi, L., Chen, F., Dong, Y., Kong, Y., Wu, Y. \& Fan, C. Ultrasensitive and selective detection of nicotinamide adenine dinucleotide by target-triggered ligationrolling circle amplification. Chem. Commun. 48, 3354-3356 (2012).

3 Zhou, L., Ou, L. J., Chu, X., Shen, G. L. \& Yu, R. Q. Aptamer-based rolling circle amplification: a platform for electrochemical detection of protein. Anal. Chem. 79, 7492-7500 (2007)

4 Shlyahovsky, B., Li, D., Weizmann, Y., Nowarski, R., Kotler, M. \& Willner, I. Spotlighting of cocaine by an autonomous aptamer-based machine. J. Am. Chem. Soc. 129, 3814-3815 (2007)

5 Notomi, T., Okayama, H., Masubuchi, H., Yonekawa, T., Watanabe, K., Amino, N. \& Hase, T. Loop-mediated isothermal amplification of DNA. Nucleic Acids Res. 28 e63 (2000).

6 Liang, C., Chu, Y., Cheng, S., Wu, H., Kajiyama, T., Kambara, H. \& Zhou, G. Multiplex loop-mediated isothermal amplification detection by sequence-based barcodes coupled with nicking endonuclease-mediated pyrosequencing. Anal. Chem. 84 3758-3763 (2012).

7 Li, J. J., Chu, Y., Lee, B. Y. H. \& Xie, X. S. Enzymatic signal amplification of molecular beacons for sensitive DNA detection. Nucleic Acids Res. 36, e36 (2008).

8 Zhao, Y., Chen, F., Wu, Y., Dong, Y. \& Fan, C. Highly sensitive fluorescence assay of DNA methyltransferase activity via methylation-sensitive cleavage coupled with nicking enzyme-assisted signal amplification. Biosens. Bioelectron. 42, 56-61 (2013).

9 Huang, Y., Chen, J., Zhao, S., Shi, M., Chen, Z. F. \& Liang, H. Label-free colorimetric aptasensor based on nicking enzyme assisted signal amplification and DNAzyme amplification for highly sensitive detection of protein. Anal. Chem. 85, 4423-4430 (2013).

10 Van Ness, J., Van Ness, L. K. \& Galas, D. J. Isothermal reactions for the amplification of oligonucleotides. Proc. Natl. Acad. Sci. USA 100, 4504-4509 (2003).

11 Liu, Y. Q., Zhang, M., Yin, B. C. \& Ye, B. C. Attomolar ultrasensitive MicroRNA detection by DNA-scaffolded silver-nanocluster probe based on isothermal amplification. Anal. Chem. 84, 5165-5169 (2012).

12 Wang, G. L. \& Zhang, C. Sensitive detection of microRNAs with hairpin probe-based circular exponential amplification assay. Anal. Chem. 84, 7037-7042 (2012).

13 Zhang, Y., Hu, J. \& Zhang, C. Y. Sensitive detection of transcription factors by isothermal exponential amplification-based colorimetric assay. Anal. Chem. 84, 9544-9549 (2012)

14 Tan, E., Erwin, B., Dames, S., Ferguson, T., Buechel, M., Irvine, B., Voelkerding, K. \& Niemz, A Specific versus nonspecific isothermal DNA amplification through thermophilic polymerase and nicking enzyme activities. Biochemistry 47, 9987-9999 (2008).

$15 \mathrm{Li}$, Y., Zeng, Y., Ji, X., Li, X. \& Ren, R. Cascade signal amplification for sensitive detection of cancer cell based on self-assembly of DNA scaffold and rolling circle amplification. Sens. Actuators B Chem. 171, 361-366 (2012). 
16 Ji, H., Yan, F., Lei, J. \& Ju, H. Ultrasensitive electrochemical detection of nucleic acids by template enhanced hybridization followed with rolling circle amplification. Anal. Chem. 84, 7166-7171 (2012).

17 Wen, Y., Xu, Y., Mao, X., Wei, Y., Song, H., Chen, N., Huang, Q., Fan, C. \& Li, D. DNAzyme-based rolling-circle amplification DNA machine for ultrasensitive analysis of MicroRNA in Drosophila Larva. Anal. Chem. 84, 7664-7669 (2012).

18 Orbach, R., Mostinski, L., Wang, F. \& Willner, I. Nucleic acid driven DNA machineries synthesizing Mg2+-dependent DNAzymes: an interplay between DNA sensing and logicgate operations. Chem. Eur. J. 18, 14689-14694 (2012).

19 Wang, F., Freage, L., Orbach, R. \& Willner, I. Autonomous replication of nucleic acids by polymerization/nicking enzyme/DNAzyme cascades for the amplified detection of DNA and the aptamer-cocaine complex. Anal. Chem. 85, 8196-8203 (2013).

20 Zhu, G., Yang, K. \& Zhang, C. Y. Simultaneous detection of mercury (II) and silver (I) ions with picomolar sensitivity. Biosens. Bioelectron. 49, 170-175 (2013).

21 Duan, R., Zuo, X., Wang, S., Quan, X., Chen, D., Chen, Z., Jiang, L., Fan, C. \& Xia, F. Lab in a tube: ultrasensitive detection of microRNAs at the single-cell level and in breast cancer patients using quadratic isothermal amplification. J. Am. Chem. Soc. 135, 4604-4607 (2013)

22 Xu, H., Xu, P., Gao, S., Zhang, S., Zhao, X., Fan, C. \& Zuo, X. Highly sensitive recognition of $\mathrm{Pb}^{2+}$ using $\mathrm{Pb}^{2+}$ triggered exonuclease aided DNA recycling. Biosens. Bioelectron. 47, 520-523 (2013).

23 Chen, J., Zhou, X. \& Zeng, L. Enzyme-free strip biosensor for amplified detection of $\mathrm{Pb}^{2+}$ based on a catalytic DNA circuit. Chem. Commun. 49, 984-986 (2013).

24 Zhuang, J., Fu, L., Xu, M., Zhou, Q., Chen, G. \& Tang, D. DNAzyme-based magnetocontrolled electronic switch for picomolar detection of lead (II) coupling with DNAbased hybridization chain reaction. Biosens. Bioelectron. 45, 52-57 (2013).

25 Tang, S., Tong, P., Li, H., Tang, J. \& Zhang, L. Ultrasensitive electrochemical detection of $\mathrm{Pb}^{2+}$ based on rolling circle amplification and quantum dots tagging. Biosens. Bioelectron. 42, 608-611 (2012).

$26 \mathrm{Li}$, T., Dong, S. J. \& Wang, E. A lead (II)-driven DNA molecular device for turn-on fluorescence detection of lead (II) ion with high selectivity and sensitivity. J. Am. Chem. Soc. 132, 13156-13157 (2010).

27 Zhao, X. H., Kong, R. M., Zhang, X. B., Meng, H. M., Liu, W. N., Tan, W., Shen, G. L. \& Yu, R. Q. Graphene-DNAzyme based biosensor for amplified fluorescence 'Turn-On' detection of $\mathrm{Pb} 2+$ with a high selectivity. Anal. Chem. 83, 5062-5066 (2011).

28 Zhang, X. B., Wang, Z., Xing, H., Xiang, Y. \& Lu, Y. Catalytic and molecular beacons for amplified detection of metal ions and organic molecules with high sensitivity. Anal. Chem. 82, 5005-5011 (2010).

29 Li, W., Yang, Y., Chen, J., Zhang, Q., Wang, Y., Wang, F. \& Yu, C. Detection of lead (II) ions with a DNAzyme and isothermal strand displacement signal amplification. Biosens. Bioelectron. 53, 245-249 (2014).
30 Zhu, X., Lin, Z., Chen, L., Qiu, B. \& Chen, G. A sensitive and specific electrochemiluminescent sensor for lead based on DNAzyme. Chem. Commun. 40, 6050-6052 (2009).

31 Yang, X., Xu, J., Tang, X., Liu, H. \& Tian, D. A novel electrochemical DNAzyme sensor for the amplified detection of Pb2+ ions. Chem. Commun. 46, 3107-3109 (2010).

32 Pelossof, G., Tel-Vered, R. \& Willner, I. Amplified surface plasmon resonance and electrochemical detection of $\mathrm{Pb}^{2+}$ ions using the $\mathrm{Pb}^{2+}$-dependent DNAzyme and hemin/ G-quadruplex as a label. Anal. Chem. 84, 3703-3709 (2012).

33 Lan, T., Furuya, K. \& Lu, Y. A highly selective lead sensor based on a classic lead DNAzyme. Chem. Commun. 46, 3896-3898 (2010).

34 Esteller, M. \& Herman, J. G. Generating mutations but providing chemosensitivity: the role of 06-methylguanine DNA methyltransferase in human cancer. Oncogene 23, $1-8$ (2004).

35 Fuks, F., Burgers, W. A., Brehm, A., Hughes-Davies, L. \& Kouzarides, T. DNA methyltransferase Dnmt1 associates with histone deacetylase activity. Nat. Genet. 24, 88-91 (2000).

$36 \mathrm{Li}$, J., Yan, H., Wang, K., Tan, W. \& Zhou, X. Hairpin fluorescence DNA probe for realtime monitoring of DNA methylation. Anal. Chem. 79, 1050-1056 (2007).

37 Tian, T., Xiao, H., Long, Y., Zhang, X., Wang, S., Zhou, X., Liu, S. \& Zhou, X. Sensitive analysis of DNA methyltransferase based on a hairpin-shaped DNAzyme. Chem. Commun. 48, 10031-10033 (2012).

$38 \mathrm{Liu}$, T., Zhao, J., Zhang, D. \& Li, G. Novel method to detect DNA methylation using gold nanoparticles coupled with enzyme-linkage reactions. Anal. Chem. $\mathbf{8 2}$, 229-233 (2010)

39 Yuan, L., Giovanni, M., Xie, J., Fan, C. \& Leong, D. Ultrasensitive IgG quantification using DNA nano-pyramids. NPG Asia Mater. 6, e112 (2014).

40 Pei, H., Zuo, X., Pan, D., Shi, J., Huang, Q. \& Fan, C. Scaffolded biosensors with designed DNA nanostructures. NPG Asia Mater. 5, e51 (2013).

(c) (i) () $\Theta$ This work is licensed under a Creative Commons Attribution-NonCommercial-NoDerivs 3.0 Unported License. The images or other third party material in this article are included in the article's Creative Commons license, unless indicated otherwise in the credit line; if the material is not included under the Creative Commons license, users will need to obtain permission from the license holder to reproduce the material. To view a copy of this license, visit http://creativecommons.org/licenses/by-nc-nd/3.0/

Supplementary Information accompanies the paper on the NPG Asia Materials website (http://www.nature.com/am) 